\title{
Disorders of Arousal
}

\author{
Andrea Galbiati $^{1} \cdot$ Raffaele Manni $^{2} \cdot$ Michele Terzaghi $^{2} \cdot$ Fabrizio Rinaldi $^{1,3}$. \\ Marco Zucconi ${ }^{1}$
}

Published online: 22 April 2016

(C) Springer International Publishing AG 2016

\begin{abstract}
Sleepwalking, sleep terrors, and confusional arousals are non-REM sleep parasomnias grouped under the specific category of disorders of arousal (DOA). These conditions are characterized by incomplete arousals from deep sleep that lead to a broad variety of emotional and motor behaviors. The advent of electroencephalography (EEG) and the discovery of rapid eye movement (REM) sleep as opposed to nonREM sleep allowed researchers to consider these phenomena from a neurophysiological rather than a purely psychopathological perspective. The utilization of new neurophysiologic techniques such as high-density scalp EEG, stereo-EEG, and advanced signal analysis methods shed further light on the mechanisms underpinning DOA and supported the notion that they should be viewed within the frame of the so-called dissociate state of being as intermediate state between wake and NREM sleep. Furthermore, other important issues like the boundaries between motor-behavioral manifestations in DOA and NFLE, neurocognitive functions, the occurrence of mentation/dream imagery during episodes in DOA and DOA daytime consequences, and treatment are still matters of debate.
\end{abstract}

This article is part of the Topical Collection on Parasomnias

Marco Zucconi

zucconi.marco@hsr.it

1 Department of Clinical Neurosciences, Neurology_Sleep Disorders Center, University San Raffaele, Scientific Institute San Raffaele, Milan, Italy

2 Unit of Sleep Medicine and Epilepsy, C. Mondino National Neurological Institute, Pavia, Italy

3 Neurology Clinic, Spedali Civili di Brescia Hospital, University of Brescia, Brescia, Italy
Keywords Disorders of arousal · Parasomnias · Electroencephalography $\cdot$ Differential diagnosis

\section{Introduction}

\section{ICDS-3: from NREM Parasomnias to DOA Concept}

Undesirable phenomena occurring during the transition from wake into sleep, during sleep, or during arousal from sleep are known as parasomnia. When these experiences manifest themselves during non-rapid eye movement (NREM) sleep, we can list them as disorders of arousal (DOA) or NREM parasomnia. According to the 3rd International Classification of Sleep Disorder (ICSD-3), this group of disorders includes confusional arousals, sleepwalking, and sleep terrors that result from incomplete arousals from deep sleep [1•]. Another NREM parasomnia, sleep-related eating syndrome (SRED) is included in the ICSD-3 but not under DOA category.

In ICSD-3, the concept of DOA has gained a more operational meaning in comparison to ICSD-2 [2] since all three conditions share a common diagnostic criterion. Accordingly, criterion A for each of the three abovementioned conditions established that "The disorder meets general criteria for NREM disorders of arousal" that in specific are as follows: recurrent episodes of incomplete awakening from sleep; inappropriate or absent responsiveness to efforts of others to intervene or redirect the person during the episode; limited (e.g., a single visual scene) or no associated cognition or dream imagery; partial or complete amnesia for the episode; and the disturbance is not better explained by another sleep disorder, mental disorder, medical condition, medication, or substance use. Furthermore, the occurrence of the episodes during the first third of the major sleep episode and the patient's 
disorientation and confusion that persist several minutes or longer following the episode are supportive criteria.

Anyway, besides these commonalities, every DOA is characterized by specific criteria (see Table 1).

Notably, according to ICSD-3 for none of these disorders, polysomnography (PSG) recording is mandatory, although it permits to rule out other sleep disorders. Despite PSG is not routinely used for the evaluation of DOA, its employment, in particular time-synchronized video-polysomnographic (vPSG), is fundamental in order to understand their pathophysiological mechanisms, demonstrating that DOA typically occurs during arousals from slow-wave sleep and that patients' sleep microstructure is altered in spite of a wellpreserved macrostructure [3••]. The main features of DOA are reported in Table 2.

In this review, we discuss several issues of DOA including neurophysiological, neurocognitive, and clinical aspects, paying particular attention to sleepwalking and sleep terrors that are the most discussed and investigated DOA in scientific literature.

\section{DOA: Prescientific, Psychiatric, and Neurophysiological Perspectives}

Sleepwalking (SW) and sleep terrors (ST) have been thought to be the result of a divine or diabolic possession for a long time [4]. In the nineteenth century, most scientists believed that sleepwalkers were enacting the content of their dreams with expression of intra-psychic conflicts. These ideas led physicians to believe that these phenomena were closely related to the individual's emotional dimensions and to interpret them as psychopathological disorders.

The advent of electroencephalography (EEG) and the discovery of REM as opposed to NREM sleep paved the way to more complex interpretation of these disorders leading to consider these phenomena from a neurophysiological rather than a purely psychopathological perspective. In his seminal paper in 1968, Broughton [5] documented that SW and ST were episodes distinct from REM sleep and strictly associated with arousals from slow-wave sleep (SWS) and coined the term "arousal disorders," which is still used today. On the basis of evoked potential testing during arousals from NREM and REM sleep, he hypothesized that SW and ST were related to abnormal cerebral reactivity during arousals from SWS. The author admitted that intra-psychic conflicts could play a role in inducing sleep changes underlying SW and ST, but stressed that there was no evidence to suggest that such conflicts per se caused these disorders. This notion was then confirmed in clinical/polysomnographic studies in SW/ST patients [6] and is considered a milestone that paved the way to the modern conceptualization of DOA as disorders of incomplete awakening and, more recently, dissociate state of being [7], which was confirmed by an ictal functional neuroimaging study [8•] and an in-depth electrophysiological brain study [9••], both demonstrating the coexistence of different local sleep and arousal states within the brain during DOA episodes.

\section{Clinical Semiology: What We Know and What We Have to Learn About}

Considering clinical semiology of DOA, one of the most debated aspects concerns the occurrence of mentation during the episode and its subsequent recall.

The occurrence of mentation/dream imagery during episodes of DOA and its relationship with DOA clinical manifestations has been a neglected matter for a long time with some authors either denying existence of dream imagery during DOA based on lack of recall by patients recovering from DOA episodes [10] or making no mention of it at all [6]. Furthermore, in case of patients' recall, most experts in the field found it difficult to establish the extent to which recall mirrored inner slow-wave sleep-related mentation or instead also resulted from what the patients experienced during the ictal and postictal phase of the episode, when he/she is in an intermediate state between wakefulness and sleep. Still, negative findings concerning dream imagery and recall are considered probative diagnostic criteria for DOA in ICSD-3 edition $[1 \bullet]$.

Table 1 Diagnostic criteria according to ICSD-3 [1•]

\begin{tabular}{llc}
\hline Confusional arousals & Sleepwalking & Sleep terrors \\
\hline $\begin{array}{l}\text { A. The disorder meets general criteria } \\
\text { for NREM disorders of arousal. }\end{array}$ & $\begin{array}{c}\text { A. The disorder meets general criteria } \\
\text { for NREM disorders of arousal. }\end{array}$ & $\begin{array}{c}\text { A. The disorder meets general criteria for } \\
\text { NREM disorders of arousal. }\end{array}$ \\
$\begin{array}{l}\text { B. The episodes are characterized by mental } \\
\text { confusion or confused behavior that occurs } \\
\text { while the patient is in bed. }\end{array}$ & $\begin{array}{c}\text { B. The arousals are associated with } \\
\text { ambulation and other complex } \\
\text { behaviors out of bed. }\end{array}$ & $\begin{array}{c}\text { B. The arousals are characterized by episodes of } \\
\text { abrupt terror, typically beginning with an }\end{array}$ \\
$\begin{array}{ll}\text { C. There is an absence of terror or ambulation } \\
\text { autside of the bed. }\end{array}$ & $\begin{array}{l}\text { alarming vocalization such as a frightening scream. } \\
\text { C. There are intense fear and signs of autonomic arousal, } \\
\text { including mydriasis, tachycardia, tachypnea, and } \\
\text { diaphoresis during an episode. }\end{array}$
\end{tabular}

For confusional arousal criteria, A-C must be met, for sleepwalking A-B, and for sleep terrors A-C 


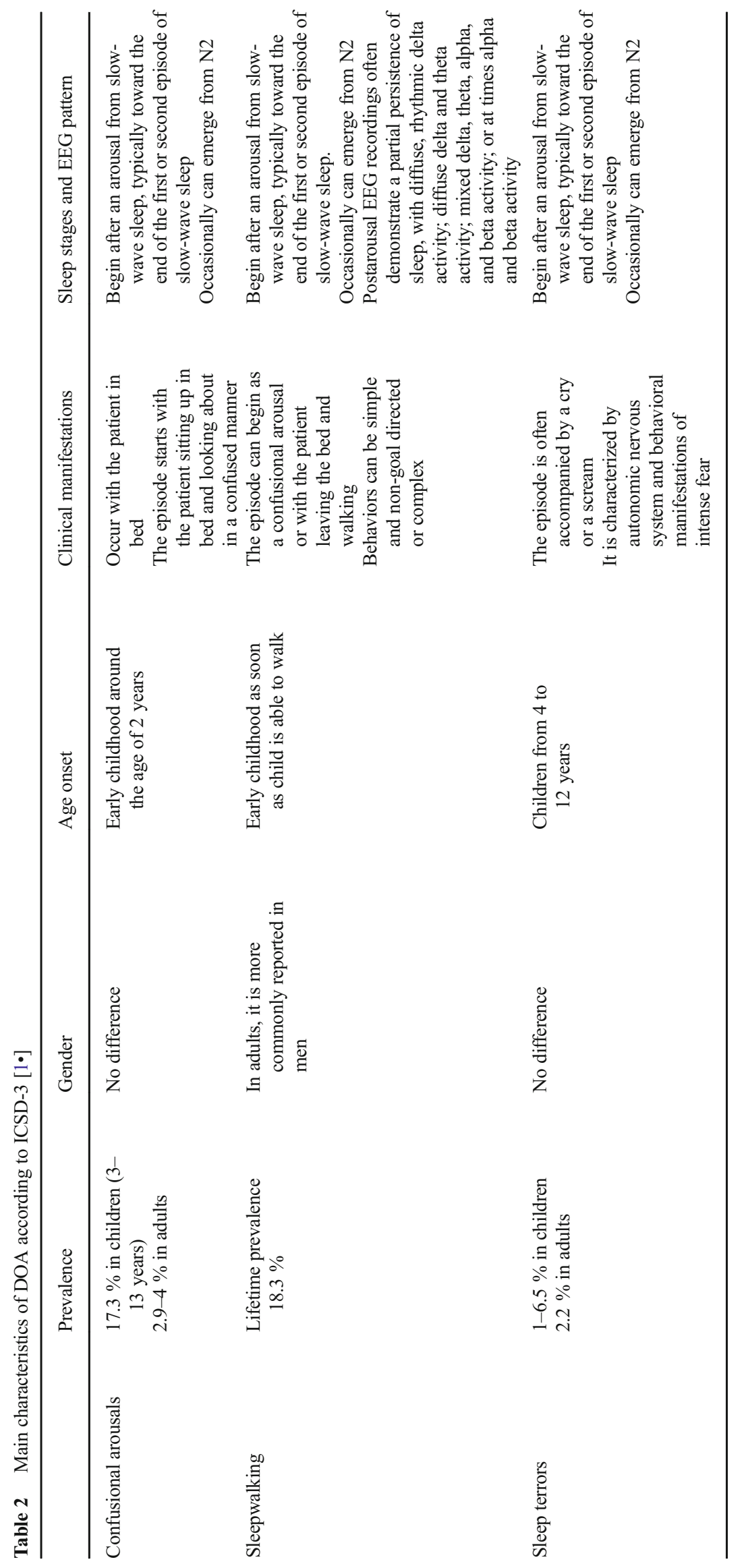


The topic of mentation during DOA episodes (particularly SW and ST episodes) has been repeatedly addressed by Arnulf's group of research in recent years with relevant insights about significance of dream mentation in these disorders $[11 \bullet, 12]$.

In fact, unpleasant dream-like mentation, mainly characterized by episodes of misfortune and feelings of apprehension, was reportedly associated with episodes of SW/ST in $71 \%$ of 38 patients at a careful questioning of the patients and more distinct patterns of dream mentation were documented in DOA as opposed to REM sleep behavior disorder (RBD) episodes [11•]. The authors hypothesize that dream mentation reflect the content of physiological dreaming in NREM in DOA and REM sleep in RBD and interpret the different enacted behaviors observed in SW and RBD (respectively, flight and fight) within the framework of the threat simulation theory of dreaming [12].

From a clinical point of view, these papers give evidence that systematic, scrupulous questioning of patients with DOA reveals that a recall in DOA episodes is less infrequent than previously thought and that mental activity associated with the episodes is peculiar and related to episodes motor-behavioral patterns.

Unusual symptoms such as chocking and hallucinatory feeling of swallowing along with symptoms of emotional distress have been recently reported in cases of DOA [13] at a systematic clinical/video-polysomnographic investigation of 68 cases of SW and ST. The frequency of occurrence of such symptoms proved to be significantly higher than that found in OSA patients. Symptoms of chocking may be part of epileptic insular seizure semiology. To be aware, these symptoms may occur in DOA and are of importance with respect to differential diagnosis between DOA, OSA, and nocturnal epileptic seizures which are episodes difficult to be distinguished.

\section{Neurophysiological and Theoretical Outlines}

Neurophysiological findings indicate that dysfunction of slow-wave sleep can be found in DOA.

The occurrence of slow-wave sleep instability is supported by a consistent number of findings [3••], and recent focus was given to slow-wave activity dynamics in DOA, in terms of organization of sleep $[14,15 \cdot]$ and specifically in relation to DOA episodes $[14,16 \bullet]$. These analyses in adult sleepwalkers confirmed that DOA were featured by enhancement of slowwave activity, but failed to delineate the true functional significance of this change. Indeed, DOA episodes were not preceded by a gradual increase in spectral power of slow-wave activity $[14,15 \cdot]$, neither conclusive findings could be drawn about acute increase in the last few seconds before episode onset $[14,15 \bullet]$.

Interestingly, when topography of different EEG bands was considered, electromagnetic tomography (eLORETA - a novel investigational tool combining spectral analysis with low resolution neuroimaging) showed significant brain changes before onset of sleepwalking not in the delta band but rather in the beta $(24$ to $30 \mathrm{~Hz}$ ) range [16•]. This study shifts the attention from slow-wave activity, reflecting the temporal EEG fluctuations of the dynamic organization of sleep to fast activity, classically reflecting the presence of arousal activity.

The search of source localization of different brain activities during DOA reflects the theoretical model of the interplay between local simultaneous sleep and wake phenomena. Various studies showed that sleep and wakefulness are neither homogenous nor mutually exclusive states [17] and that different brain activities can be found in local circuits during sleep [18••]. The local dimension of sleep in the brain shows that while certain parts of the brain may be active (awake), other parts can be simultaneously deactivated (asleep). Local activations manifest in restricted cortical areas as an interruption of sleep patterns and by the appearance of wake-like patterns. Spontaneous local activations in the motor cortex were demonstrated to be endowed in the normal physiology of NREM and REM sleep [19•, 20]. It was proposed that lower arousal threshold reflected in greater activation of the motor cortex may represent an ethological adaptation and a possible evolutionary advantage [19•]. Neurophysiological investigations proved to be able to capture these local sleep/ wake phenomena in DOA [16•, 21••, 22••]. Indeed, stereoelectroencephalography capturing confusional arousal demonstrated local activations in the motor, frontal and central cingulated, insular and temporopolar cortices, and enhancement of delta activity in the frontal and parietal association areas; these local activation were postulated to underlie typical features of DOA $[9 \bullet \bullet, 21 \bullet \bullet]$.

The conceptualization of intrinsic networks and microcircuits and their imbalance on a regional, inter-regional, and connectome-wide level has gained momentum in the last decade; in particular, it could become a key concept in epilepsy with the development of the idea of system epilepsies [23], applied to nocturnal frontal lobe epilepsy (NFLE) spectrum [24]. Analogously, DOA were suggested to result from the dysfunction of the arousal system, where dysfunctional arousal leads to imbalance of different microcircuitry linked together, thus leading to the clinical expression of DOA [25, 26]. A challenge that should help to clarify the pathophysiology of DOA is represented by our ability to understand the origin and the modulating factors of dysfunctional arousal.

Disturbed timing of thalamo-cortical oscillations has been suggested to play a central role in the generation of arousal system disorder [25], and cingulated cortex seems to play a crucial function $[9 \bullet \bullet, 16 \bullet, 21 \bullet \bullet$. In particular, arousal/ antiarousal twin systems driven by antagonistic thalamocortical activity involved in NREM sleep and wakefulness were suggested to be foremost factors in the genesis of DOA [25]. Wavelet spectral analyses showed that thalamic activity 
during arousals is clearly stereotyped and characterized by a decrease in all frequency bands [27]. Consistently, during DOA, the ventro-medial portion of the thalamus showed the appearance of fast activity super imposable to the thalamic activity recorded during wakefulness [22••]. Conversely, patterns of cortical activity during arousals are heterogeneous (despite heterogeneity is reduced during N3 sleep), and their spectral structure is influenced by homeostatic pressure, sleep macrostructure, specific cortical areas, and arousal modality (spontaneous versus nociceptive) [27]. Thus, stereotyped thalamic arousal is associated with different patterns of cortical arousal, hence reinforcing the concept that knowledge of factors modulating arousal activity can be essential in understanding the pathophysiogenesis of DOA.

\section{Neuroimaging in DOA}

Despite the growing interest and utilization of neuroimaging techniques, to the best of our knowledge, only few studies have investigated DOA with such methodology [28]. In a single case study, Bassetti et al. [8•] investigated with ${ }^{99 \mathrm{~m}} \mathrm{Tc}$ ECD SPECT a 16-year-old man affected by sleepwalking during an episode. An increment of regional cerebral blood flow was observed during the episode in comparison with quiet SWS in the anterior cerebellum and in the posterior cingulate cortex while the dorsolateral prefrontal cortex, mesial frontal cortex, and left angular gyrus were deactivated during sleepwalking. This deactivation can be responsible of the lack of awareness that is a typical feature of these episodes; vice versa, the persistent activation of the cingulate cortex could explain the motor behaviors. Taken together, this evidence highlights the dissociation between awareness and motor activity.

Recently, another study employed ${ }^{99 \mathrm{~m}}$ Tc-ECD SPECT in order to investigate brain structures associated with somnambulism [29]. Ten sleepwalkers and 10 healthy controls were recruited and scanned after a night of sleep. Furthermore, 8 of these patients and 9 of the controls were scanned after a night of total sleep deprivation. After a night of total sleep deprivation, a significant lower regional brain perfusion, localized in the inferior temporal gyrus, was found in SW compared to controls. Sleep deprivation is known to cause episodes of somnambulism, but these results underline also its importance in altering brain functionality during wakefulness in these patients. Although very interesting, this result cannot elucidate whether these abnormalities represent a prodromal sign for the development of SW episodes or a consequence of sleep deprivation.

Regarding sleep terrors, a structural MRI investigation was reported [30]. An increased T2 signal from the right thalamus ascribable to a low-grade tumor was found. The authors suggested that an adulthood onset of sleep terror can be symptomatic of a neuropathological condition and has to be investigated carefully. Hence, a thalamic dysfunction would be able to influence the arousal system and contribute in provoking sleep terrors.

Scientific literature investigating DOA by means of neuroimaging is relatively scarce. Further studies are needed in order to replicate these findings in larger samples.

\section{Neurocognitive Functions in DOA}

The majority of the works regarding DOA has been focused on PSG- and EEG-related variables. Only a small number of studies investigated cognitive functions in DOA. Therefore, little is known about daytime performance in patients affected by these conditions. Previous works have focused their attention in defining personality traits, mainly in sleepwalkers, suggesting an increased anxiety and neuroticism in this condition [31, 32]. Recently, Perogamvros et al. [33] evaluated rewardrelated personality trait in sleepwalkers by means of the Temperament and Character Inventory (TCI) [34]. The authors' hypothesis is that exploratory behaviors during NREM sleep parasomnias could be linked to the activation of the mesolimbic dopaminergic (ML-DA) network. Accordingly, exploratory behaviors are frequent during sleepwalking episodes and are often modulated by dreamlike experiences [35]. Pergamvros et al. found high scores in novelty seeking (NS) subscale of the TCI suggesting an elevated impulsivity. This could reflect an increased activity in ML-DA pathway during wake time that extends during sleep causing overt behaviors. Furthermore, NS1 correlated with REM sleep percentage. This positive correlation would reflect a link between dopamine, REM sleep, and dream-like activity.

Another study by Labelle et al. [36•] was aimed at evaluating neurocognitive function in patients affected by SW. In this investigation, sustained attention and executive functions were tested by means of the continuous performance test (CPT) and the Stroop color-word test in the morning after a PSG assessment and 1 week later after $25 \mathrm{~h}$ of sleep deprivation. During normal wakefulness, sleepwalkers did not show any impairment in comparison to healthy controls in neuropsychological tests. Therefore, these patients present wellpreserved cognitive performances (psychomotor speed, alertness, and sustained attention) after nocturnal sleep. Vice versa, after sleep deprivation, results are different. Within group differences were observed on alertness and vigilance that decrease after sleep deprivation in comparison to normal wakefulness in both control subjects and sleepwalkers. Interestingly, in the sleep deprivation condition, sleepwalkers made more errors in the flexibility condition of the Stroop task and more commission errors on the CPT in comparison to control subjects. These variables are known to be linked to behavioral impulsivity and attentional dyscontrol [37]. Therefore, the authors suggested that sleep deprivation in 
sleepwalkers affects the control of inhibition and foster motor impulsivity.

A typical hallmark of DOA is SWS instability. As SWS activity is known to be strongly associated to memory consolidation [38], a possible negative effect of these disorders on memory function is arguable. Uguccioni et al. [39] tested verbal memory consolidation during sleep in patients affected by SW and ST. Two verbal memory tasks were employed in order to assess memory consolidation across the night. Furthermore, a learning task was used during the day to test consolidation after $9 \mathrm{~h}$ of wakefulness. Despite patients exhibited an increased number of awakenings form SWS and a longer wake time period after sleep onset in comparison to control, no significant differences were found in task performance between the groups. Both patients and controls' performances worsened during daytime in comparison to nighttime sleep. Accordingly, Oudiette at al. [40] found that a procedural task was well consolidated after night-time sleep in a group of SW and ST patients. Surprisingly, they reported the case of a sleepwalker who re-enacted the motor sequence of the learned task during an episode. On the contrary, in the study of Uguccioni et al. [39], 7 patients showed episode of sleeptalking during the recording nights but the content of the sentences was not related to the list of words of the task.

Since the exiguity of studies investigating neurocognitive function in DOA, further investigations are needed in order to shed light on the effect of these disorders on daytime performances. Furthermore, as reported by the aforementioned studies, this line of research could be helpful in clarifying some pathophysiological aspects of DOA.

\section{Comorbidities and Differential Diagnosis}

In addition to sleep disorders such as restless legs syndrome with periodic limb movements during sleep (PLMS), obstructive sleep apnea or other upper airway obstructions with sleep fragmentation, other disorders such as psychiatric disorders, misuse/abuse of psychotropic drugs (neuroleptics, hypnotics, antihistamines, antidepressants, etc.), and alcohol abuse were reportedly comorbid in DOA [3••]. Despite the classical picture of DOA without excessive daytime sleepiness, impairment of daytime functioning with abnormal sleep propensity exemplified by the multiple sleep latency test (MSLT) and/or at the Epworth sleepiness scale (ESS), independent from the number of DOA episodes during the night, has been documented in DOA patients. The slow-wave sleep fragmentation and the instability of sleep microstructure as measured by cyclic alternating pattern (CAP) have been indicated as possible causes [41•], without excluding the daytime functional involvement of the cerebral cortex demonstrated by transcranial magnetic stimulation abnormalities [42].

The dream-like mentation, typical of REM parasomnias as REM sleep behavior disorder (RBD), has been also suggested to be part of the somnambulistic episode in many patients, indicating a possible interaction between dream and motor activity also in DOA, instead of the classical characteristic of NREM parasomnia phenomenon thought to occur without concomitant dream activity or complex mental contents $[11 \bullet$, 43•].

To distinguish DOA from NFLE may be challenging. Clinical interview as well as ad hoc questionnaires such as the FLEP scale [44] proved to be of limited value [45] in quite a few cases. Furthermore, a personal or familial history of DOA is common in patients with NFLE, making shared pathophysiological pathways plausible in these conditions and sometimes making the characterization of the nocturnal episodes more difficult [46, 47]. Thus video-EEG polysomnography is frequently needed to get a definite diagnosis (see Fig. 1). However, the boundaries between hyperkinetic seizures in NFLE, motor manifestations in parasomnias, and physiological movements during NREM sleep may remain uncertain despite the use surface EEGPSG and even stereo-EEG recordings in a number of cases. It is well known that stereotypic tonic-dystonic movements are typical and distinctive of NFLE. However, similar motor patterns have recently been reported in DOA. Complex automatisms similar to those observed in sleep-related seizures have been documented in patients with non-epileptic episodes $[22 \bullet \bullet]$. This would mean that some particular features of motor activation resulting in effective emotional and motor patterns can be observed without a clear EEG "epileptic" activation. These manifestations have been hypothesized to be under the control of a specific central pattern generator (CPG), which can be activated and triggered by different sleep patterns (including sleep instability with arousals oscillation as in the cyclic alternating pattern) as well as by EEG epileptiform discharges. So far, stereoEEG findings in DOA are limited to case reports. In fact, this complex investigation cannot be considered a routine methodology of functional exploration of the brain to better understand the etiology and the mechanisms of the arousal parasomnias. However, the discovery of simultaneous sleep-like and wake-like activities in different brain regions during an episode of parasomnia and the importance of local sleep and local slow-waves theory in interpreting the nature and morphology of the episodes of parasomnia may lead to a definite pathophysiologic explanation of the mechanisms underpinning DOA. The utilization of new neurophysiologic techniques as high-density scalp EEG and advanced signal analysis methods may further shed

Fig. 1 Arousal and post-arousal EEG during a confusional arousal arising from stage 3 NREM in an 8-year-old boy. The EEG shows high-voltage delta busts followed by delta/theta slow activity, intermingled with alpha activity and superimposed muscular artifacts. The pattern is followed by stage 2 of NREM sleep 
a

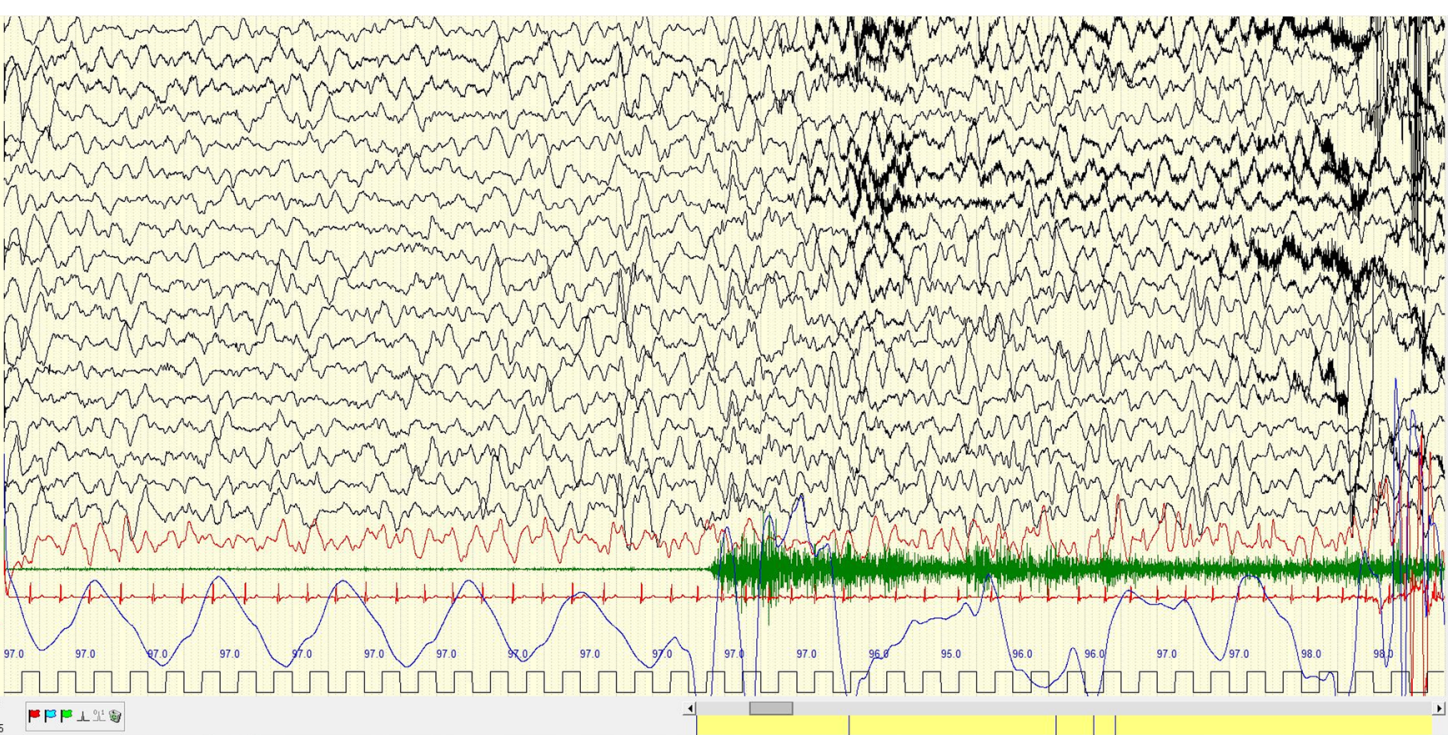

b

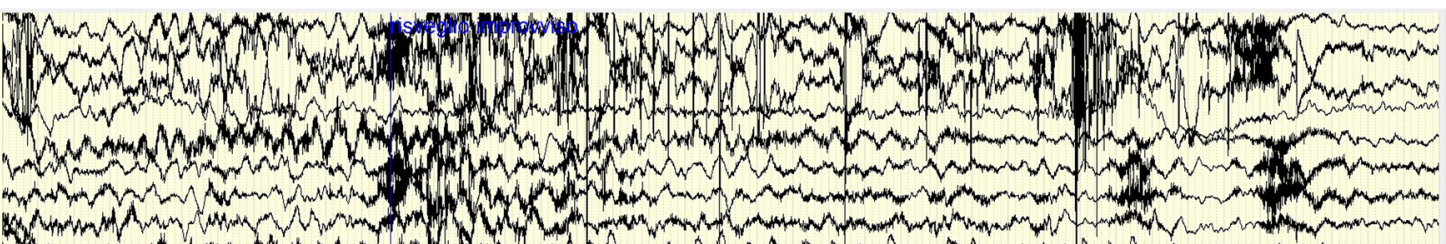

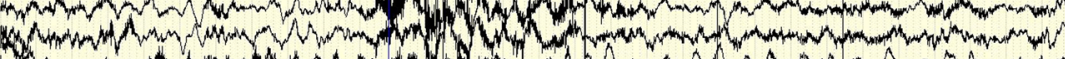
A monch Wan Don Xom

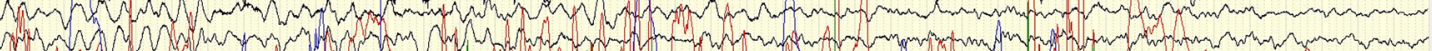

MLO+ MLOTHOR- THOR+

SPO2-SPO2+ 980

MKR+ MKR.
Timer $00: 01: 08: 0$

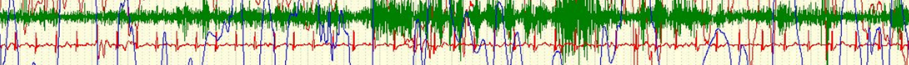

C

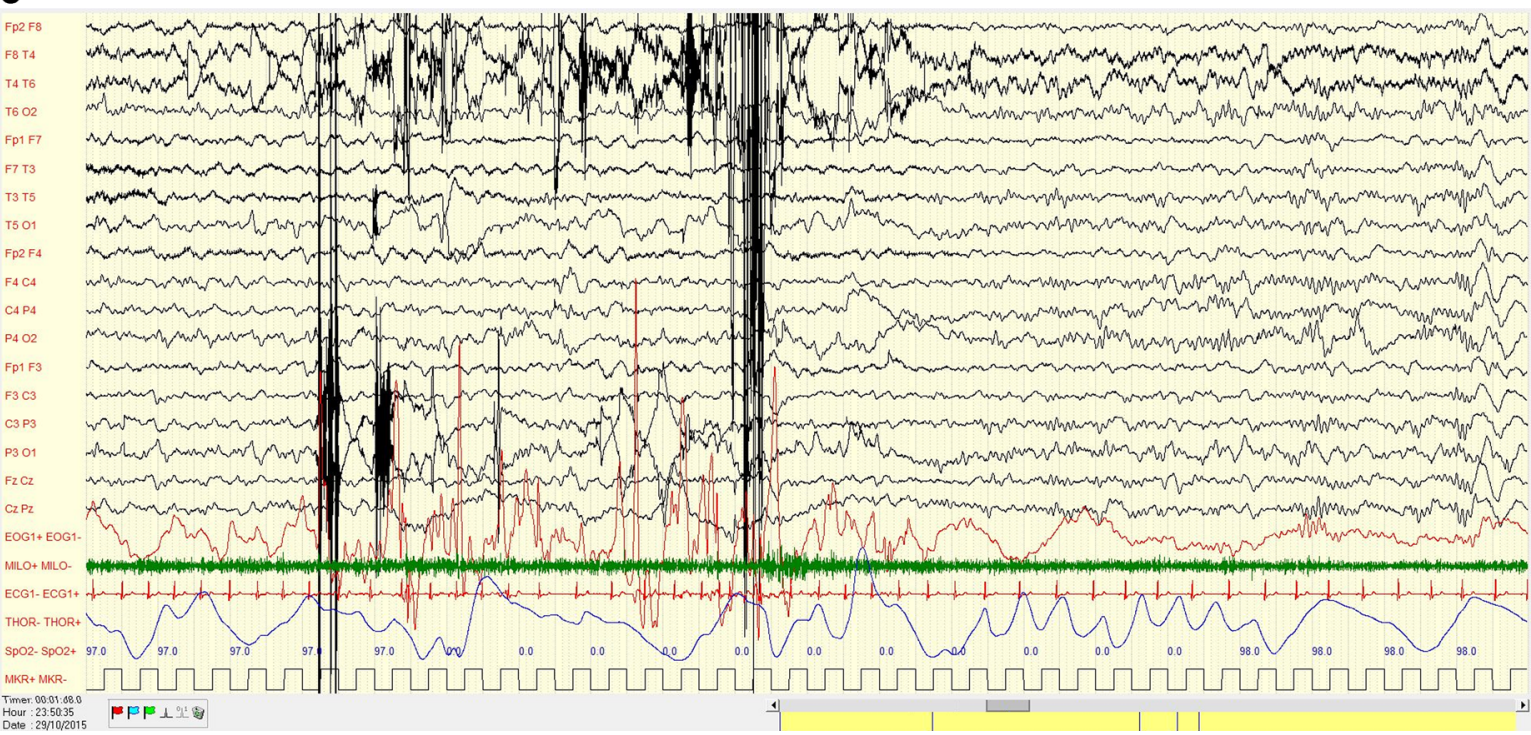


light in this still shadow fascinating mechanism of the sleep disorders and the relationship with the sleep physiology [48].

\section{Treatment}

\section{Confusional Arousals}

The primary therapy for all parasomnias is adequate patient and bed partner education. In fact, the disease course is usually benign and tends to resolve spontaneously with time. Both the patient and bed partner should be educated about safety precautions for the bedroom environment, aimed at reducing potential sources of the injury. The partner should avoid to awaken the patient during an episode, as this can possibly trigger violent behavior. A trial of sleep extension or scheduled awakening may be considered. SBD were reported to frequently occur in children with parasomnias with SBD mimicking parasomnia episodes in some cases and triggering them in others [49]. Also, PLMS, RLS/WED, and gastroesophageal reflux can determine arousals from sleep; therefore, specific treatments will reduce the parasomnias.

There is no specific pharmacological management for confusional arousals. However, when symptoms become persistent and severe, benzodiazepine (clonazepam [50]) or antidepressants (imipramine and clomipramine [50]) have been suggested to be effective.

Scheduled or anticipatory awakening have been suggested as a behavioral technique to prevent these events [51•].

\section{Sleepwalking}

First-line treatment of sleepwalking is supportive, and aims to create a safe environment and to avoid precipitants such as sleep deprivation, gastroesophageal reflux disease, and alcohol ingestion. Looking for possible triggering factor such as comorbid sleep disordered breathing is of utmost importance, since their treatment can resolve sleepwalking as well [52].
Parents should be reassured about the drop of prevalence of these disorders with age and that SW is not linked with underlying psychiatric illness.

A number of non-pharmacologic treatments, including anticipatory awakening, psychotherapy, and hypnotherapy, have been proposed [52]. If events are frequent and disruptive and persist into adulthood, pharmacological approaches should be considered [53]. Benzodiazepine is usually the first-line choice (diazepam and clonazepam [54-56], triazolam [57], flurazepam [53]). Other agents are antidepressants such as tricyclic antidepressants, such as serotonin-selective reuptake inhibitors (SSRIs) (paroxetine [58], imipramine [59]). Reports of successful treatment with low dose quetiapine have also been reported [60]. A recent retrospective survey assessed the efficacy of treatments of DOA that presented as SW in 39, ST in 28, CA in 9, and more than one type of DOA or mixed parasomnia in 27. The study reported good efficacy of treatment with clonazepam in $73.7 \%$ of cases, describing also response to treatment with sertraline, clomipramine, and temazepam in sporadic cases, as well as with cognitive behavioral therapy (CBT) [61].

Nevertheless, the pharmacological treatment of sleepwalking remains largely anecdotal [62], and the evidence supporting the efficacy of treatment is scarce. In a study by Guilleminault et al., four out of five subjects placed on clonazepam had limited results at 3 months follow-up, reporting only a decrease in the frequency of sleepwalking events under particularly high daytime stress [63]. Cases of remission after treatment with olanzapine [64] and biperiden [65] have been reported. With no high-quality evidence to support recommendations for treatments of somnambulism, a patienttailored approach is recommended.

\section{Sleep Terrors}

The first approach in children with infrequent episodes of ST is reassurance, since they often resolve spontaneously with time. As with the other disorders of arousals, patients should

Table 3 Pharmacological therapies for DOA

\begin{tabular}{|c|c|c|}
\hline Confusional arousals & Sleepwalking & Sleep terrors \\
\hline $\begin{array}{l}\text { - Benzodiazepines (e.g., } \\
\text { clonazepam [50]) } \\
\text { - Antidepressants (e.g., } \\
\text { imipramine, } \\
\text { clomipramine [50]) }\end{array}$ & $\begin{array}{l}\text { - Benzodiazepines (e.g., diazepam, } \\
\text { clonazepam [54-56], triazolam } \\
\text { [57], flurazepam [53]) } \\
\text { - Antidepressants (e.g., tricyclic } \\
\text { antidepressants, SSRIs such as } \\
\text { paroxetine [58], imipramine [59], } \\
\text { clomipramine, sertraline [61]) } \\
\text { - Antipsychotics (e. g., quetiapine } \\
\text { [60], temazepam [61], olanzapine } \\
\text { [64], and biperiden [65]) }\end{array}$ & $\begin{array}{l}\text { Children: } \\
\text { - Melatonin, L-5-hydroxytryptophan } \\
{[70 \bullet \text { }]} \\
\text { Adults: } \\
\text { • Benzodiazepines (e.g., diazepam } \\
\text { [66], clonazepam [67•]) } \\
\text { - Antidepressants (imipramine [68], } \\
\text { trazodone [69], clomipramine, } \\
\text { paroxetine [51•]) }\end{array}$ \\
\hline
\end{tabular}


be taught to avoid sleep deprivation and other trigger factors such as alcohol, stress, and drugs. Behavioral methods such as psychotherapy, relaxation therapy, autogenic training, and hypnosis can be used to prevent ST [52]. Anticipatory or scheduled awakening have also been proposed, but special attention must be paid when the patient is sleep deprived [52]. When the episodes become more frequent, self-injurious, or persistent into adulthood, pharmacological treatment is justified.

Proposed pharmacological treatment includes benzodiazepines (diazepam [66], clonazepam [67•]) and antidepressants (imipramine [68], trazodone [69] clomipramine and paroxetine $[51 \bullet])$.

In children, the first-choice treatment is usually melatonin or L-5-hydroxytryptophan [70•]. However, sleep terrors may in some cases be associated with psychological traumas or with an underlying psychopathology [71]; in this scenario, psychotherapy may be considered in addition to medication treatment.

Pharmacological therapies for DOA are reported in Table 3.

\section{Conclusions}

In ICSD-3, the concept of DOA has gained a more operational meaning in comparison to ICSD-2 [2] since all three conditions share a common diagnostic criterion and pathophysiology. Both neurophysiological and functional neuroimaging data support now the notion that DOA consist of intermediate states between wakefulness and NREM sleep, with dysfunctional arousal system and imbalance of different microcircuitry in cingulate and prefrontal and frontal cortex, playing a fundamental role in their pathogenesis. While most of the semiological features of DOA remain peculiar and distinctive, some recently evidenced features concerning dream mentation and recall on the one hand and motor patterns on the other make differential diagnosis with REM parasomnia, NFLE, and sleep-motor disorders particularly challenging in a number of cases. Finally, no definite guidelines are there on when and how to treat DOA in their various, non-comorbid, and comorbid clinical contexts.

\section{Compliance with Ethical Standards}

Conflict of Interest Andrea Galbiati, Raffaele Manni, Michele Terzaghi, Fabrizio Rinaldi, and Marco Zucconi declare that they have no conflict of interest.

Human and Animal Rights and Informed Consent This article does not contain any studies with human or animal subjects performed by any of the authors.

\section{References}

Paper of particular of interest, published recently, have been highlighted as:

- Of importance

- Of major importance

1. American Academy of Sleep Medicine. International classification of sleep disorders. 3rd ed. Darien: American Academy of Sleep Medicine; 2014. Here are the new definitions according to the last and adjourned Classification of Sleep Disorders.

2. American Academy of Sleep Medicine. International classification of sleep disorders. 2nd ed. Westchester: American Academy of Sleep Medicine; 2005.

3.• Zadra A, Desautels A, Petit D, Montplaisir J. Somnambulism: clinical aspects and pathophysiological hypotheses. Lancet Neurol. 2013;12:285-94. A theoretical and pathophysiological paper on the DOA with complete references.

4. Umanath S, Sarezky D, Finger S. Sleepwalking through history: medicine, arts, and courts of law. J Hist Neurosci. 2011;20:253-76.

5. Broughton RJ. Sleep disorders: disorders of arousal? Science. 1968;159:1070-8.

6. Jacobson A, Kales A, Lehmann D, Zweig JR. Somnambulism: allnight electroencephalographic studies. Science. 1965;148:975-7.

7. Mahowald MW, Cramer Bornemann MA, Schenck CH. State dissociation, human behavior, and consciousness. Curr Top Med Chem. 2011;11:2392-402.

8. Bassetti C, Vella S, Donati F, Wielepp P, Weder B. SPECT during sleepwalking. Lancet. 2000;356:484-5. The first document of Neuro-Imaging during an episode of SW.

9.• Terzaghi M, Sartori I, Tassi L, Rustioni V, Proserpio P, Lorusso G, et al. Dissociated local arousal states underlying essential clinical features of non-rapid eye movement arousal parasomnia: an intracerebral stereo-electroencephalographic study. J Sleep Res. 2012;21:502-6. A case report documenting dissociate state and local sleep in DOA with depth-recording.

10. Gastaut H, Broughton RA. Clinical and polygraphic study of episodic phenomena during sleep. In: Wortis J, editor. Recent advances in biological psychiatry. New York: Plenum Press; 1965. p. 197.

11. Oudiette D, Leu S, Pottier M, Buzare MA, Brion A, Arnulf I. Dreamlike mentations during sleepwalking and sleep terrors in adults. Sleep. 2009;32:1621-7. The first reporting investigating dream report in $\mathrm{SW}$ and $\mathrm{ST}$.

12. Uguccioni G, Golmard JL, de Fontréaux AN, Leu-Semenescu S, Brion A, Arnulf I. Fight or flight? Dream content during sleepwalking/sleep terrors vs. rapid eye movement sleep behavior disorder. Sleep Med. 2013;14:391-8.

13. Flamand M, Herlin B, Leu-Semenescu S, Attali V, Launois C, Arnulf I. Choking during sleep: can it be expression of arousal disorder? Sleep Med. 2015;16:1441-7.

14. Jaar O, Pilon M, Carrier J, Montplaisir J, Zadra A. Analysis of slowwave activity and slow-wave oscillations prior to somnambulism. Sleep. 2010;33:1511-6.

15. Perrault R, Carrier J, Desautels A, Montplaisir J, Zadra A. Electroencephalographic slow waves prior to sleepwalking episodes. Sleep Med. 2014;15:1468-72. Report of Neurophysiological EEG (Slow wave activity) correlates before starting DOA episodes.

16. Januszko P, Niemcewicz S, Gajda T, Wołyńczyk-Gmaj D, Piotrowska AJ, Gmaj B, et al. Sleepwalking episodes are preceded by arousal-related activation in the cingulate motor area: EEG current density imaging. Clin Neurophysiol. 2016;127:530-6. The first report of cingulate area activation preceding $\mathrm{SW}$ by high density EEG data. 
17. Vyazovskiy VV, Olcese U, Hanlon EC, Nir Y, Cirelli C, Tononi G. Local sleep in awake rats. Nature. 2011;472:443-7.

18.• Nir Y, Staba RJ, Andrillon T, Vyazovskiy VV, Cirelli C, Fried I, et al. Regional slow waves and spindles in human sleep. Neuron. 2011;70:153-69. Basic article on the relationship among local sleep in different cortical areas.

19. Nobili L, Ferrara M, Moroni F, De Gennaro L, Russo GL, Campus $\mathrm{C}$, et al. Dissociated wake-like and sleep-like electro-cortical activity during sleep. Neuroimage. 2011;58:612-9. Demonstration of local sleep in different cortical areas in humans.

20. De Carli F, Proserpio P, Morrone E, Sartori I, Ferrara M, Gibbs SA, et al. Activation of the motor cortex during phasic rapid eye movement sleep. Ann Neurol. 2016;79:326-30.

21.• Terzaghi M, Sartori I, Tassi L, Didato G, Rustioni V, LoRusso G, et al. Evidence of dissociated arousal states during NREM parasomnia from an intracerebral neurophysiological study. Sleep. 2009;32:409-12. Evidence of different state of "being" and dissociated arousals by deep electrode studies.

22.• Gibbs SA, Proserpio P, Terzaghi M, Pigorini A, Sarasso S, Lo Russo G, et al. Sleep-related epileptic behaviors and non-REMrelated parasomnias: insights from stereo-EEG. Sleep Med Rev. 2016;25:4-20. Review on the different approach to epileptic and non-epileptic behaviors during sleep by means of deep electrode recording.

23. Van Diessen E, Diederen SJH, Braun KPJ, Jansen FE, Stam CJ. Functional and structural brain networks in epilepsy: what have we learned? Epilepsia. 2013;54:1855-65.

24. Halász P, Kelemen A, Szücs A. The role of NREM sleep microarousals in absence epilepsy and in nocturnal frontal lobe epilepsy. Epilepsy Res. 2013;107:9-19.

25. Halasz P, Kelemen A, Szücs A. Physiopathogenetic interrelationship between nocturnal frontal lobe epilepsy and NREM arousal parasomnias. Epilepsy Res Treat. 2012;2012:312693.

26. Terzaghi M, Manni R. Mapping the features of arousal parasonmnias in adults: on the way to better understand arousal parasomnias and ease differential diagnosis. Sleep Med. 2015;16: 1439-40.

27. Peter-Derex L, Magnin M, Bastuji H. Heterogeneity of arousals in human sleep: a stereo-electroencephalographic study. Neuroimage. 2015;123:229-44

28. Dang-Vu TT, Desseilles M, Petit D, Mazza S, Montplaisir J, Maquet P. Neuroimaging in sleep medicine. Sleep Med. 2007;8: 349-72.

29. Dang-Vu TT, Zadra A, Labelle MA, Petit D, Soucy JP, Montplaisir J. Sleep deprivation reveals altered brain perfusion patterns in somnambulism. PLoS One. 2014;10, e0133474.

30. Di Gennaro G, Autret A, Mascia A, Onorati P, Sebastiano F, Quarato P. Night terrors associated with thalamic lesion. Clin Neurophysiol. 2004;115:2489-92.

31. Ohayon MM, Guilleminault C, Priest RG. Night terrors, sleepwalking, and confusional arousals in the general population: their frequency and relationship to other sleep and mental disorders. J Clin Psychiatry. 1999;60:268-76.

32. Brion A, Flamand M, Oudiette D, Voillery D, Golmard JL, Arnulf I. Sleep-related eating disorder versus sleepwalking: a controlled study. Sleep Med. 2012;13:1094-101.

33. Perogamvros L, Aberg K, Gex-Fabry M, Perrig S, Cloninger CR, Schwartz S. Increased reward-related behaviors during sleep and wakefulness in sleepwalking and idiopathic nightmares. PLoS One. 2015;10:e0134504.

34. Cloninger CR. A unified biosocial theory of personality and its role in the development of anxiety states. Psychiatr Dev. 1986;4:167226.

35. Zadra A, Pilon M, Montplaisir J. Phenomenology of somnambulism. Sleep. 2006;29:A269.
36. Labelle MA, Dang-Vu TT, Petit D, Desautels A, Montplaisir J, Zadra A. Sleep deprivation impairs inhibitory control during wakefulness in adult sleepwalkers. J Sleep Res. 2015;24:658-65. The impact of sleep deprivation as provoking factor on DOA episodes in adult $\mathrm{SW}$.

37. Sørensen L, Plessen KJ, Adolfsdottir S, Lundervold AJ. The specificity of the Stroop interference score of errors to ADHD in boys. Child Neuropsychol. 2014;20:677-91.

38. Fogel S, Martin N, Lafortune M, Barakat M, Debas K, Laventure S, et al. NREM sleep oscillations and brain plasticity in aging. Front Neurol. 2012;3:176.

39. Uguccioni G, Pallanca O, Golmard JL, Leu-Semenescu S, Arnulf I Is sleep-related verbal memory consolidation impaired in sleepwalkers? J Sleep Res. 2015;24:197-205.

40. Oudiette D, Constantinescu I, Leclair-Visonneau L, Vidailhet M, Schwartz S, Arnulf I. Evidence for the re-enactment of a recently learned behavior during sleepwalking. PLoS One. 2011;6, e18056.

41. Montplaisir J, Petit D, Pilon M, Mongrain V, Zadra A. Does sleepwalking impair daytime vigilance? J Clin Sleep Med. 2011;7:219. The theory and review on possible mechanisms underlying daytime sleepiness in DOA patients.

42. Oliviero A, Della Marca G, Tonali PA, Pilato F, Saturno E, Dileone $\mathrm{M}$, et al. Functional involvement of cerebral cortex in adult sleepwalking. J Neurol. 2007;254:1066-72.

43. Manni R, Terzaghi M. Dreaming and enacting dreams in nonrapid eye movement and rapid eye movement parasomnia: a step toward a unifying view within distinct patterns? Sleep Med. 2013;14:3878. An editorial on mental activity and dreaming activity in REM and NREM parasomnias.

44. Derry CP, Davey M, Johns M, Kron K, Glencross D, Marini C, et al. Distinguishing sleep disorders from seizures: diagnosing bumps in the night. Arch Neurol. 2006;63:705-9.

45. Manni R, Terzaghi M, Repetto A. The FLEP scale in diagnosing nocturnal frontal lobe epilepsy, NREM and REM parasomnias: data from a tertiary sleep and epilepsy unit. Epilepsia. 2008;49:1581-5.

46. Tinuper P, Provini F, Bisulli F, Vignatelli L, Plazzi G, Vetrugno R, et al. Movement disorders in sleep: guidelines for differentiating epileptic from non-epileptic motor phenomena arising from sleep. Sleep Med Rev. 2007;11:255-67.

47. Bisulli F, Vignatelli L, Naldi I, Licchetta L, Provini F, Plazzi G, et al. Increased frequency of arousal parasomnias in families with nocturnal frontal lobe epilepsy: a common mechanism? Epilepsia. 2010;51:1852-60.

48. Lustenberger C, Huber R. High density electroencephalography in sleep research: potential, problems, future perspective. Front Neurol. 2012;3:77.

49. Guilleminault C, Palombini L, Pelayo R, Chervin RD. Sleepwalking and sleep terrors in prepubertal children: what triggers them? Pediatrics. 2003;111:17-25.

50. Mahowald MW, Schenck CH. Non-rapid eye movement sleep parasomnias. Neurol Clin. 2005;23:1077-106.

51. Attarian H. Treatment options for parasomnias. Neurol Clin. 2010;28:1089-106. A review on DOA treatments.

52. Galbiati A, Rinaldi F, Giora E, Ferini-Strambi L, Marelli S Behavioural and cognitive-behavioural treatments of parasomnias. Behav Neurol. 2015. doi:10.1155/2015/786928.

53. Mendelson WB. Hypnotics: basic mechanisms and pharmacology. In: Kryger MH, Roth T, Dement WC, editors. Principles and practice of sleep medicine. 3rd ed. Philadelphia: W. B. Saunders; 2000. p. 407-13.

54. Goldbloom D, Chouinard G. Clonazepam in the treatment of neuroleptic induced somnambulism. Am J Psychiatry. 1984;141:1486.

55. Schenck CH, Milner DM, Hurwitz TD, Bundlie SR, Mahowald MW. A polysomnographic and clinical report on sleep-related injury in 100 adult patients. Am J Psychiatry. 1989;146:1166-73. 
56. Schenck CH, Mahowald MA. Long-term nightly benzodiazepine treatment of injurious parasomnias and other disorders of disrupted nocturnal sleep in 170 adults. Am J Med. 1996;100:333-7.

57. Berlin RM, Qayyum U. Sleepwalking: diagnosis and treatment through the life cycle. Psychosomatics. 1986;27:755-60.

58. Lillywhite AR, Wilson SJ, Nutt DJ. Successful treatment of night terrors and somnambulism with paroxetine. Br J Psychiatry. 1994;164:551-4.

59. Marshall JR. The treatment of night terrors associated with the posttraumatic syndrome. Am J Psychiatry. 1975;132:293-5.

60. Gill JS, Pillai SK, Koh OH, Jambunathan ST. Low dose quetiapine in the treatment of an adolescent with somnambulism: a case report. Acta Neurol Belg. 2011;111:155-6.

61. Attarian H, Zhu L. Treatment options for disorders of arousal: a case series. Int J Neurosci. 2013;123:623-5.

62. Remulla A, Guilleminault C. Arousal parasomnias. In: Guilleminault C, editor. Handbook of clinical neurophysiology volume, clinical neurophysiology of sleep disorders. Amsterdam: Elsevier; 2005.

63. Guilleminault C, Kirisoglu C, Bao G, Arias V, Chan A, Li KK. Adult chronic sleepwalking and its treatment based on polysomnography. Brain. 2005;128:1062-9.
64. Faridhosseini F, Zamani A. A case report of somnambulism associated with olanzapine. Iran J Psych Behav Sci. 2012;6: $72-4$.

65. Hodoba D, Schmidt D. Biperiden for treatment of somnambulism in adolescents and adults with or without epilepsy: clinical observations. Epilepsy Behav. 2012;25:517-28.

66. Allen RM. Attenuation of drug-induced anxiety dreams and pavor nocturnus by benzodiazepines. J Clin Psychiatry. 1983;44:106-8.

67. Cochen De Cock V. Sleepwalking. Curr Treat Options Neurol. 2016;18:6. An adjourned review on SW treatment.

68. Burstein A, Burstein A. Treatment of night terrors with imipramine. J Clin Psychiatry. 1983;44:82.

69. Balone R. Sleep terror disorder and insomnia treated with trazodone: a case report. Ann Clin Psychiatry. 1994;6:161-3.

70. Bruni O, Ferri R, Miano S, Verrillo E. L-5-Hydroxytryptophan treatment of sleep terrors in children. Eur J Pediatr. 2004;163: 402-7. One of the few studies comparing pharmacologic treatment for DOA vs Placebo in children.

71. Fisher C, Byrne J, Edwards A, Kahn E. A psychophysiological study of nightmares. J Am Psychoanal Assoc. 1970;18:747-82. 